Pacific Journal of Mathematics

FAITHFUL DISTRIBUTIVE MODULES OVER INCIDENT 


\title{
FAITHFUL DISTRIBUTIVE MODULES OVER INCIDENCE ALGEBRAS
}

\author{
ROBERT B. FEINBERG
}

Let $Q$ be a lower finite quasi-ordered set and let $I(Q)$ be the incidence algebra of $Q$ over a field $K$. In this paper we determine all faithful distributive modules over $I(Q)$ and relate the result to the structure of the outer automorphism group of the algebra. In the case when $Q$ is finite we also determine all left ideals $L$ of $I(Q)$ such that ${ }_{I(Q)} I(Q) / L$ is a faithful distributive module over $I(Q)$.

In the abstract characterization of incidence algebras it is necessary to consider the existence and structure of faithful distributive modules over them. In $\S 1$ we determine up to isomorphism all faithful distributive modules over an incidence algebra. In $\S 2$ we relate this result to the structure of the outer automorphism group of the algebra. In $\S 3$ we consider the incidence algebra of a finite quasi-ordered set and determine for it all left ideals $L$ with the property that the quotient module determined by $L$ is a faithful distributive module over the algebra.

Let $Q$ be a locally finite quasi-ordered (q.o.) set, i.e., $Q$ has a relation $r$ which is reflexive and transitive and for which every segment $[x, y]=\{z \in Q ; x r z r y\}$ is finite. The incidence algebra $I(Q)$ of $Q$ over a field $K$ is the (associative unital) algebra of functions $f: Q \times Q \rightarrow K$ with the property that $f(x, y) \neq 0 \Rightarrow x r y$, under the product

$$
f^{*} g(x, y)=\sum_{x r z r y} f(x, z) g(z, y) .
$$

The unit $\delta$ of $I(Q)$ is defined by

$$
\begin{array}{rlll}
\delta(x, y)=1 & \text { if } & x=y \\
=0 & \text { if } & x \neq y
\end{array} .
$$

For $x r y \in Q$, define $\delta_{x y} \in I(Q)$ by

$$
\begin{aligned}
\delta_{x y}(u, v)=1 & & \text { if } & x=u, y=v \\
& =0 & & \text { otherwise. }
\end{aligned}
$$

and set $e_{x}=\delta_{x x}$.

The standard topology on $I(Q)$ is defined by stipulating that a net $\left\{f_{\lambda}\right\}_{\lambda \in \theta} \subseteq I(Q)$ converges to $f \in I(Q)$ iff $\forall x r y \in Q, f_{\lambda}(x, y)=f(x, y)$ eventually, i.e., $\exists \mu \in \theta$ such that $\forall \mu^{\prime} \geqq \mu, f_{\mu^{\prime}}(x, y)=f(x, y)$. It is 
easy to verify that $I(Q)$ equipped with the above topology is a topological algebra, when the field $K$ has the discrete topology. When $Q$ is finite, the standard topology on $I(Q)$ reduces to the discrete topology.

We now introduce the following equivalence relation on $Q: x \sim$ $y$ iff $x r y$ and $y r x$. Let $\bar{x}$ denote the $\sim$ equivalence class of $x \in Q$ and let $\hat{Q}$ denote the collection of all equivalence classes. Then $\hat{Q}$ is a partially ordered (p.o.) set under the relation $\leqq$ defined by $\bar{x} \leqq \bar{y}$ iff $x r y$. Using the these notions it is convenient at this time to present the following two technical results on $I(Q)$, in the case when $Q$ is a finite q.o. set.

LEmma $0.1([2, \S 2],[5, \S 1])$. The maximal ideals of $I(Q)$ are all subspaces of the form $J_{\bar{x}}=\left\{f \in I(Q) ; f\left(x_{1}, x_{2}\right)=0 \quad \forall x_{1}, x_{2} \in Q\right.$ with $\left.\bar{x}_{1}=\bar{x}_{2}=\bar{x}\right\}$, where $\bar{x}$ is some element of $\hat{Q}$. Further, assuming $\bar{x}=\left\{x_{i}\right\}_{i=1}^{n}$ and letting $K_{n}$ denote the (simple) algebra of $n \times n$ matrices over the field $K$, we have that the map $\pi_{x}^{-}: I(Q) \rightarrow K_{x}$ defined by $\left(\pi_{x}^{-} f\right)_{u v}=$ $f\left(x_{u}, x_{v}\right), 1 \leqq u, v \leqq n$, is an epimorphism with kernel $J_{\bar{x}}$, so that $I(Q) / J_{\bar{x}} \simeq K_{n}$.

We will use $|S|$ to denote the cardinality of a set $S$.

LEMMA $0.2([2, \S 1])$. An element $f$ of $I(Q)$ is invertible in $I(Q)$ iff $\pi_{\bar{x}}(f)$ is invertible in $K_{|\bar{x}|}, \forall \bar{x} \in Q$.

Now let $A$ be a topological algebra and let $M$ be a (unital left) module over $A$. If the action of $A$ on $M$ is continuous in $A$ when $M$ has the discrete topology, we shall say that $M$ is topologically compatible (with $A$ ). If $M$ has a distributive lattice of submodules, and is faithful and topologically compatible, we shall say that $M$ is a faithful distributive module (over $A$ ). The following three results on faithful distributive modules over incidence algebras are from $[5, \S 2]$.

Lemma 0.3. Let $Q$ be locally finite. Then $I(Q)$ has a faithful distributive module iff $Q$ is lower finite.

We will explicitly construct such modules in the course of proving Theorem 1.1, to follow.

Lemma 0.4. Let $M$ be a topologically compatible, faithful module over $I(Q)$. Then $M$ is faithful distributive iff $e_{x} \cdot M$ is 1-dimensional $\forall x \in Q$. In particular, when $Q$ is a finite quasi-ordered set of cardinality $n$, a faithful module $M$ is faithful distributive iff 
$\operatorname{dim} M=n$.

Lemma 0.5. Let $M$ be a faithful distributive module over $I(Q)$. For each $z \in Q$, chose $m_{z} \in e_{z} \cdot M \backslash\{0\}$. Then $B=\left\{m_{z}\right\}_{z \in Q}$ is a basis for $M$, and for $w r x \in Q, \delta_{w x} \cdot m_{x}=\alpha(w, x) m_{w}$, for some $\alpha(w, x) \in K \backslash\{0\}$.

1. Faithful distributive modules. In this section we determine up to isomorphism all faithful distributive mondules over an incidence algebra. We begin with some new notions. An element $f \in I(Q)$ is multiplicative if (i) $f(x, y) \neq 0 \forall x r y \in Q$ (ii) $f(x, y) f(y, z)=$ $f(x, z) \forall x r y r z \in Q$. It is easy to show that the collection of multiplicative elements forms an abelian group under the pointwise product, $f g(x, y)=f(x, y) g(x, y)$. We shall denote this group by $Z(Q)$. The identity $\zeta$ is given by $\zeta(x, y)=1, \forall x r y$ in $Q$ and the inverse $\tilde{f}$ of $f \in Z(Q)$ is given by $\tilde{f}(x, y)=[f(x, y)]^{-1}$. It follows from (i) and (ii) above that for all $f \in Z(Q), f(x, x)=1 \forall x \in Q$.

Given any $\alpha: Q \rightarrow K \backslash\{0\}$, we define $d_{\alpha} \in Z(Q)$ by $d_{\alpha}(x, y)=\alpha(x) \alpha(y)^{-1}$. Let $B(Q)=\left\{d_{\alpha} \in Z(Q)\right\}$. Then $B(Q)$ is a (necessarily normal) subgroup of $Z(Q)$, and thus we may construct the quotient group $H(Q)=$ $Z(Q) / B(Q)$. It may be shown, for example that $H(Q) \simeq K$ for the quasi-ordered set $\left\{x_{1}, x_{2}, x_{3}, x_{4}\right\}$, where $x_{1} r x_{3}, x_{1} r x_{4}, x_{2} r x_{3}, x_{2} r x_{4}$. On the other hand, $H(Q)=0$ if $Q$ has an element $z$ comparable to all others. For if $\sigma \in Z(Q)$, we may define $\alpha: Q \rightarrow K \backslash\{0\}$ by

$$
\begin{array}{rll}
\alpha(w)=1, & \text { if } & w=z \\
\sigma(z, w), & \text { if } & z r w \\
\sigma(w, z)^{-1}, & \text { if } & w r z
\end{array}
$$

Then $\alpha$ is well-defined and $\sigma=d_{\alpha}$ as may be verified, so that $\sigma \in B(Q)$.

We can now present our main result.

THEOREM 1.1. If $Q$ is a lower finite q.o. set, then there is a bijective correspondence between the elements of $H(Q)$ and the isomorphism classes of faithful distributive modules over $I(Q)$.

Proof. For any $\nu \in Z(Q)$, let $M_{\nu}$ be the $K$-vector space with basis $\{z\}_{z \in Q}$. For $f \in I(Q)$ and $x \in Q$ (considered as a basis element of $M_{\nu}$ ), set

$$
f \cdot{ }_{\nu} x=\sum_{w r x} f(w, x) \nu(w, x) w .
$$

Then ${ }_{\nu}$ is well-defined, by lower finiteness of $Q$, and the fact that $\nu$ is multiplicative implies that for all $g \in I(Q),\left(f^{*} g\right) \cdot{ }_{\nu} x=f \cdot{ }_{\nu}\left(g \cdot{ }_{\nu} x\right)$. Now extend by linearity to define an action , of $I(Q)$ on $M_{\nu}$. It 
is readily verified that this action makes $M_{\nu}$ a faithful module over $I(Q)$. Further, for each $x \in Q, e_{x} \cdot{ }_{\nu} M_{\nu}=K x$, so that $e_{x} \cdot{ }_{\nu} M_{\nu}$ is 1 dimensional. It is easy to see that $M_{\nu}$ is topologically compatible with $I(Q)$. Hence by Lemma $0.4, M_{\nu}$ is a faithful distributive module over $I(Q)$.

In the case when $\nu=\zeta$ and $Q$ is finite, of cardinality $n, M_{\nu}$ affords the canonical representation of $I(Q)$ as an algebra of $n \times n$ matrices over $K$. See [5, §2] or [6] for details.

Suppose now that $\nu, \tau \in Z(Q)$ and that $\tau B(Q)=\nu B(Q)$. We show that $M_{\nu}$ and $M_{\tau}$ are isomorphic $I(Q)$-modules. Note that $\tau \tilde{\nu} \in B(Q)$, so that $\exists \alpha: Q \rightarrow K \backslash\{0\}$ such that $\tau \tilde{\nu}=d_{\alpha}$, i.e., for all $x r y \in Q$, $\tau(x, y) \nu(x, y)^{-1}=d_{\alpha}(x, y)=\alpha(x) \alpha(y)^{-1}$, or $\tau(x, y) \alpha(y)=\alpha(x) \nu(x, y)$. Define $\varphi: M_{\nu} \rightarrow M_{\tau}$ by setting $\varphi(x)=\alpha(x) x$, for $x \in Q$, and extend by linearity to a map on $M$. Then $\varphi$ is a linear bijection. Note also that for $f \in I(Q)$ and $x \in M_{\nu}$,

$$
\begin{aligned}
\varphi\left(f_{\nu} \cdot x\right) & =\varphi \sum_{w r x} f(w, x) \nu(w, x) w \\
& =\sum_{w r x} f(w, x) \nu(w, x) \alpha(w) w,
\end{aligned}
$$

while

$$
f \cdot{ }_{\tau} \varphi(x)=f \cdot{ }_{\tau} \alpha(x) x=\sum_{w r x} f(w, x) \tau(w, x) \alpha(x) w .
$$

It follows that $\varphi\left(f \cdot{ }_{\nu} x\right)=f \cdot{ }_{\tau} \varphi(x)$, so that $\varphi$ preserves module action as well. Hence $\varphi$ is a module isomorphism.

Conversely, suppose that $\nu, \tau \in Z(Q)$ and that $M_{\nu}$ and $M_{\tau}$ are isomorphic $I(Q)$-modules. We show that $\tau B(Q)=\nu B(Q)$. Let $\varphi$ : $M_{\nu} \rightarrow M_{\tau}$ be a module isomorphism. Since $\nu$ is multiplicative, $\nu(x, x)=$ 1, $\forall x \in Q$. Hence $\varphi(x)=\varphi[\nu(x, x) x]=\varphi\left(e_{x}{ }_{\tau} x\right)=e_{x}{ }_{\tau}{ }_{\tau} \varphi(x)$. This implies that $\varphi(x)=\alpha(x) x$, for some $\alpha(x) \in K \backslash\{0\}$. Now for $w r x \in Q$, $\varphi\left(\delta_{w x} \cdot x\right)=\varphi[\nu(w, x) w]=\nu(w, x) \varphi(w)=\nu(w, x) \alpha(w) w$, while also

$$
\varphi\left(\delta_{w x} \cdot{ }_{\nu} x\right)=\delta_{w x} \cdot{ }_{\tau} \varphi(x)=\delta_{w x} \cdot{ }_{\tau} \alpha(x) x=\tau(w, x) \alpha(x) w .
$$

This implies that $\nu(w, x) \alpha(w)=\tau(w, x) \alpha(x)$, or equivalently, $\tau \widetilde{\mathcal{\nu}}(w, x)=$ $d_{\alpha}(w, x)$. Hence $\tau \widetilde{\nu}=d_{\alpha} \in B(Q)$, so that $\tau B(Q)=\nu B(Q)$.

Now define $\xi: H(Q) \rightarrow$ isomorphism classes of faithful distributive $I(Q)$-modules $\}$ by $\xi[\nu B(Q)]=$ isomorphism class of $M_{\nu}$. The above results show that $\xi$ is well-defined and $1-1$. We show that $\xi$ is onto. Let $M$ be any faithful distributive module over $I(Q)$. For each $z \in Q$, choose $m_{z} \in e_{z} \cdot M \backslash\{0\}$. Then by Lemma $0.5, B=\left\{m_{z}\right\}_{z \in Q}$ is a basis for $M$, and for $w r x$ in $Q, \delta_{w x} \cdot m_{x}=\alpha(w, x) m_{w}$, some $\alpha(w, x) \in K \backslash\{0\}$. For wrxry $\in Q$ we have $\alpha(w, y) m_{w}=\delta_{w y} \cdot m_{y}=\delta_{w x} * \delta_{x y} \cdot m_{y}=\delta_{w x} \alpha(x, y) m_{x}=$ $\alpha(w, x) \alpha(x, y) m_{w}$. Hence, $\alpha$ is multiplicative, considered as an element 
of $I(Q)$. It follows readily that $M$ and $M_{\alpha}$ are isomorphic as $I(Q)$ modules, via the map sending $m_{x} \in M$ to $x \in M_{\alpha}$. Thus $\xi[\alpha B(Q)]$ is the module isomorphism class of $M$, showing that $\xi$ is onto.

COROLlaRY 1.1.1. Let $Q$ be a lower finite q.o. set. Then $I(Q)$ has a unique isomorphism class of faithful distributive modules iff $H(Q)=0$.

These results may be dualized in a straightforward way to the consideration of isomorphism classes of cofaithful right comodules over the incidence coalgebra $C(Q)$. See [5] for more details on these notions.

As a simple application of the results in this section, let $Q_{1}$ be the quasi-ordered set $\left\{x_{\imath}\right\}_{i=1}^{n}$, where $x_{i} r x_{j}, 1 \leqq i \leqq j \leqq n$. Then $I\left(Q_{1}\right)$ is isomorphic to $T_{n}$, the algebra of $n \times n$ upper triangular matrices over $K$, and the faithful distributive modules over $I(Q)$ are exactly the faithful modules of dimension $n$, by Lemma 0.4. It then follows directly from Corollary 1.1.1 that $I\left(Q_{1}\right) \simeq T_{n}$ has a unique isomorphism class of faithful modules of dimension $n$. Note that each such module affords the canonical $n$-dimensional representation of $T_{n}$. As another application, let $Q_{2}$ be the quasi-ordered set $\left\{y_{i}\right\}_{i=1}^{n}$, where $y_{i} r y_{j}, 1 \leqq i, j \leqq n$. Then $I\left(Q_{2}\right)$ is isomorphic to $K_{n}$, the algebra of $n \times n$ matrices over $K$, and the faithful distributive modules over $I\left(Q_{2}\right)$ are exactly the faithful irreducible modules. It then follows directly from Corollary 1.1.1 that $I\left(Q_{2}\right) \simeq K_{n}$ has a unique isomorphism class of faithful irreducible (= primitive) modules.

2. Automorphisms. In this section we study the automorphisms of an incidence algebra, relating our results to those of the last section. We restrict ourselves to topological automorphisms of $I(Q)$, i.e., automorphisms which preserve topological structure, and we let Aut $I(Q)$ denote the group of all such automorphisms of $I(Q)$, where $Q$ is a locally finite q.o. set.

We begin by discussing three classes of (topological) automorphisms of $I(Q)$.

1. For $f \in I(Q), f$ invertible, the inner automorphism of $I(Q)$ with respect to $f$ is given by $I_{f}(g)=f^{*} g^{*} f^{-1}$. Let Inn $I(Q)$ denote the group of all such automorphisms. Then $\operatorname{Inn} I(Q)$ is a normal subgroup of Aut $I(Q)$.

2. For $\nu \in I(Q), \nu$ multiplicative, there is an automorphism $L_{\nu}$ of $I(Q)$ defined by $L_{\nu}(f)=\nu f$. Let $\mathscr{L}[I(Q)]$ denote the group of all such automorphisms. Then $\operatorname{Inn} I(Q) \cap \mathscr{L}[I(Q)]=\left\{L_{\alpha} \in[I(Q)] ; \alpha \in B(Q)\right\}$ 
as is shown in [1].

3. Let Aut $Q$ denote the automorphism group of $Q$, i.e., the collection of order-preserving bijections. For $\kappa \in$ Aut $Q$ there is an automorphism $C_{\kappa}$ of $I(Q)$ defined by $C_{\kappa} f(x, y)=f(\kappa x, \kappa y)$.

We now examine the structure of Aut $Q$ more closely. For $x \in Q$, let $\bar{x}$ be its $\sim$ equivalence class, as defined in $\S 0$, and let $\widehat{Q}$ denote the p.o. set of equivalence classes of $Q$. It is easy to see that there is a canonical embedding of Aut $Q$ into Aut $\hat{Q}$ given by $\varphi \rightarrow \hat{\varphi}$, where $\hat{\varphi}(\bar{x})=\overline{\varphi x}$. Further, the image of Aut $Q$ under this embedding, denoted $\overline{\text { Aut } Q}$, consists of all $\psi \in$ Aut $\hat{Q}$ such that $|\psi(\bar{x})|=$ $|\bar{x}|, \forall \bar{x} \in Q$.

We can now state the following result, which completely describes the structure of Aut $I(Q)$.

THEOREM 2.1. Assume $\theta \in$ Aut $I(Q)$, where $Q$ is locally finite. Then $\theta=I_{f} \circ L_{\nu} \circ C_{k}$, for suitably chosen $I_{f} \in \operatorname{Inn} I(Q), L_{\nu} \in \mathscr{L}[(Q)]$, and $\kappa \in$ Aut $Q$. Further, $\kappa$ is uniquely determined up to its image in Aut $\hat{Q}$.

This theorem is proven by Baclawski [1], assuming that $Q$ is a p.o. set, and consequently without topological restrictions on Aut $I(Q)$. The proof for the more general case is a straightforward generalization of Baclawski's proof, and utilizes knowledge about the maximal closed ideals of $I(Q)$ as well as the fact that every automorphism of $K_{n}$ is inner. See [4., Ch. 7] for details.

Now let Out $I(Q)=$ Aut $I(Q) / \operatorname{Inn} I(Q)$, the outer automorphism group of $I(Q)$. The following corollary is an easy consequence of Theorem 2.1, along with our knowledge of $\operatorname{Inn} I(Q) \cap \mathscr{L}[I(Q)]$.

Corollary 2.1.1. Out $I(Q) \simeq \overline{\operatorname{Aut}(Q)}$ iff $H(Q)=0$.

In particular, Out $I(Q) \simeq \overline{\operatorname{Aut}(Q)}$ in the case when $Q$ is connected and has an element above or below all other elements. This latter result is obtained by different means in [7].

The concluding corollary of this section utilizes Corollary 2.1.1, and provides a purely algebraic relation between the results of the last section and of this one.

COROLlary 2.1.2. I(Q) has a unique isomorphism class of faithful distributive modules iff Out $I(Q) \simeq \overline{\operatorname{Aut}(Q)}$. 
3. Faithful distributive left ideals. In this section we study left ideals $L$ of $I(Q)$ such that ${ }_{I(Q)} I(Q) / L$ is a faithful distributive module over $I(Q)$. Such left ideals occur only when $Q$ is finite, and we begin by working towards a proof of this fact.

The first result needed is readily established.

LEMma 3.1. Let $R$ be associative ring with unit and let $M$ be a module over $R$. Then $M$ is cyclic iff $\exists$ a left ideal $L$ of $R$ such that $M \simeq{ }_{R} R / L$, where ${ }_{R} R$ denotes $R$ regarded as a left module over itself and $\simeq$ denotes left $R$-module isomorphism. Further, in the case when $M$ is cyclic with generator $m$, we may take $L$ to be $\{\alpha \in A$; $\alpha \cdot m=0\}$.

Before continuing, recall that if $Q$ is a locally finite q.o. set, then $I(Q)$ has a faithful distributive module iff $Q$ is lower finite (Lemma 0.3).

LEMMA 3.2. Let $Q$ be a lower finite q.o. set and let $M$ be any faithful distributive module over $I(Q)$ which is cyclic, with generator $m$. If

$$
m=\sum_{i=1}^{n} a_{\imath} m_{z_{i}}, m_{z_{i}} \in e_{z_{i}} \cdot M \backslash\{0\}, a_{\imath} \in K \backslash\{0\},
$$

then every maximal element $x$ of $Q$ must be $\sim$ equivalent to at least one $z_{\imath}, 1 \leqq i \leqq n$.

Proof. Suppose there is a maximal element $x$ of $Q$ such that $\bar{x} \neq \bar{z}_{\imath}, 1 \leqq i \leqq n$. Since $m$ is a generator of $M, m_{x}=f \cdot m$, for some $f \in I(Q)$. Hence $m_{x}=e_{x} \cdot m_{x}=\left(e_{x}^{*} f\right) \cdot m$. Now since $x$ is a maximal element of $Q$,

$$
e_{x}^{*} f=\sum_{x^{\prime} \in \tilde{x}} f\left(x, x^{\prime}\right) \delta_{x x^{\prime}} .
$$

It follows then that $\left(e_{x}^{*} f\right) \cdot m=0$, since for all $x^{\prime} \in \bar{x}, x^{\prime} \notin\left\{z_{i}\right\}_{i=1}^{n}$. From this contradiction we obtain that any maximal element $x$ of $Q$ is $\sim$ equivalent to at least one of $z_{1}, \cdots, z_{n}$.

COROLLARY 3.2.1. Let $Q$ be lower finite. If $I(Q)$ has a faithful distributive module which is cyclic, then $Q$ is finite.

Proof. By Lemma 3.2 there is a finite set $\left\{z_{i}\right\}_{i=1}^{n} \subseteq Q$ such that every maximal element is $\sim$ equivalent to at least one $z_{i}, 1 \leqq i \leqq n$. Since $Q$ is locally finite, the $\sim$ equivalence class of any element is finite. Thus $Q$ has only finitely many maximal elements, say $\left\{x_{j}\right\}_{j=1}^{d}$. 
Now $Q=\bigcup_{j=1}^{d}\left\{y ; y r x_{j}\right\}$, and since $Q$ is lower finite, each set $\left\{y ; y r x_{j}\right\}$ is finite. Therefore $Q$ is finite.

Lemma 3.3. Let $Q$ be a finite quasi-ordered set. Let $M$ be a faithful distributive module over $I(Q)$ and let $B=\left\{m_{z}\right\}_{z \in Q}$ be a basis for $M$, as described in Lemma 0.5. Set

$$
m=\sum_{j=1}^{d} m_{x_{j}},
$$

where $\left\{x_{j}\right\}_{j=1}^{d}$ is the collection of maximal elements of $Q$. Then $m$ is a generator of $M$.

Proof. For every $y \in Q \exists$ maximal element $x_{i}$ such that $y r x_{i}$. We then have that

$$
\delta_{y x_{i}} \cdot \sum_{j=1}^{d} m_{x_{j}}=\delta_{y_{i}} \cdot m_{x_{i}}=\alpha_{y x_{i}} m_{y},
$$

for some $\alpha_{y_{i}} \in K \backslash\{0\}$. This shows that $I(Q) \cdot m=M$, so that $m$ is a generator of $M$.

We can now establish the fact cited at the beginning of the section.

Lemma 3.4. Let $Q$ be a lower finite quasi-ordered set. The following are equivalent.

(1) $Q$ is finite.

(2) $I(Q)$ has a left ideal $L$ such that ${ }_{I(Q)} I(Q) / L$ is a faithful distributive module over $I(Q)$.

(3) $I(Q)$ has a faithful distributive module which is cyclic.

(4) Every faithful distributive module over $I(Q)$ is cyclic.

$$
\begin{aligned}
& \text { Proof. } \quad(1) \Rightarrow \text { (4) Lemma 3.3. } \\
& \text { (4) } \Rightarrow \text { (3) Immediate. } \\
& \text { (3) } \Leftrightarrow \text { (2) Lemma 3.1. } \\
& (3) \Rightarrow(1) \text { Corollary 3.2.1. }
\end{aligned}
$$

We shall assume that all q.o. sets $Q$ are finite in the remainder of this section. Our object of study will be faithful distributive left ideals of $I(Q)$, left ideals $L$ with the property that ${ }_{I(Q)} I(Q) / L$ is a faithful distributive module over $I(Q)$. It is easy to show that an arbitrary left ideal $L^{\prime}$ is faithful distributive iff (i) $L^{\prime}$ contains no nonzero two sided ideal $I(Q)$, and (ii) the lattice of left ideals of $I(Q)$ containing $L^{\prime}$ is distributive.

We now present the main result of this section, which is es- 
sentially a determination of all faithful distributive left ideals of $I(Q)$.

THEOREM 3.5. Let $Q$ be a finite quasi-ordered set. Then given any isomorphism class $\mathscr{C l}$ of faithful distributive modules over $I(Q)$, there is a unique isomorphism class $\mathscr{L}$ of left ideals of $I(Q)$ such that for all $L \in \mathscr{L}$ and $M \in \mathscr{M},{ }_{I(Q)} I(Q) / L$ and $M$ are isomorphic $I(Q)$-modules.

Proof. Let $\left\{\bar{x}_{i}\right\}_{i=1}^{n}$ be the collection of maximal elements of $\hat{Q}$, where $\bar{x}_{i}=\left\{x_{i j}\right\}_{j=1}^{n_{i}} \subseteq Q$. It then follows from Lemmas 3.3 and 3.1 that

$$
m=\sum_{i=1}^{n} \sum_{j=1}^{n_{2}} m_{x_{i j}},
$$

is a generator of $M$, that $L=\{f \in I(Q) ; f \cdot m=0\}$ is a left ideal of $I(Q)$, and that $M \simeq{ }_{I(Q)} I(Q) / L$.

Now suppose $L^{\prime}$ is any other left ideal of $I(Q)$ such that ${ }_{I(Q)} I(Q) / L^{\prime} \simeq$ $M$, via an isomorphism $\psi$. Then $m^{\prime}=\psi\left(\delta+L^{\prime}\right)$ is a generator of $M$. Further, for all $f \in I(Q), \psi\left(f+L^{\prime}\right)=\psi\left(f\left[\delta+L^{\prime}\right]\right)=f \cdot \psi\left(\delta+L^{\prime}\right)=$ $f \cdot m^{\prime}$. Hence $f \in L^{\prime} \Leftrightarrow \psi\left(f+L^{\prime}\right)=0 \Leftrightarrow f \cdot m^{\prime}=0$, so that $L^{\prime}=\{f \in I(Q)$; $\left.f \cdot m^{\prime}=0\right\}$.

Assume

$$
m^{\prime}=\sum_{i=1}^{n} \sum_{j=1}^{n_{i}} a_{\imath j} m_{x_{i j}}+\sum_{k=1}^{h} b_{k} m_{y_{k}}
$$

where

$$
\left\{a_{i j}\right\}_{1 \leq i \leq n},\left\{b_{k}\right\}_{1 \leqq k \leqq h} \subseteq K
$$

and

$$
\left\{y_{k s}\right\}_{k=1}^{h}
$$

are the nonmaximal elements of $Q$. We know by Lemma 3.2 that for each $i \leqq n$ there is at least one $j \leqq n_{i}$ such that $a_{i j} \neq 0$.

For $1 \leqq i \leqq n, 1 \leqq j \leqq n_{i}$, let

$$
\begin{aligned}
& a_{\imath j}^{\prime}=a_{i j} \quad \text { if } \quad a_{i j} \neq 0 \\
& =1 \quad \text { if } \quad a_{i j}=0
\end{aligned}
$$

and let

$$
\begin{aligned}
& b_{\imath j}=0 \quad \text { if } \quad a_{\imath j} \neq 0 \\
& =-1 \text { if } a_{\imath j}=0 \text {. }
\end{aligned}
$$

For each $y_{k}$, choose $y_{k}^{\prime}$ maximal in $Q$ such that $y_{k} r y_{k}^{\prime}$. For $1 \leqq$ $i \leqq n, 1 \leqq j \leqq n_{i}-1$, define $c_{i j} \in K$ by

$$
\delta_{x_{i j} x_{i, j+1}} \cdot m_{x_{i, j+1}}=c_{\imath j} m_{x_{i j}} .
$$


For $1 \leqq i \leqq n$, define $c_{i, n_{i}} \in K$ by

$$
\delta_{x_{i, n_{i}} x_{i 1}} \cdot m_{x_{i 1}}=c_{i, n_{i}} m_{x_{i, n_{i}}} \cdot
$$

Finally, for $1 \leqq k \leqq h$ define $c_{k} \in K$ by

$$
\delta_{y_{k} y_{k}^{\prime}} \cdot m_{y_{k}^{\prime}}=c_{k} m_{y_{k}} \cdot
$$

Define $g \in I(Q)$ by

$$
\begin{aligned}
g= & \sum_{i=1}^{n}\left\{\sum_{j=1}^{n_{i}} a_{i j}^{\prime} e_{x_{i j}}+\sum_{j=1}^{n_{i}-1} \frac{b_{i j}}{c_{i j}} \delta_{x_{i j} x_{i}, j+1}\right. \\
& \left.+\frac{b_{i, n_{i}}}{c_{i, n_{i}}} \delta_{x_{i, n_{i}} x_{i j}}\right\}+\sum_{k=1}^{h} \frac{b_{k}}{c_{k}} \delta_{y_{k} y_{k}^{\prime}} .
\end{aligned}
$$

It is easy to verify that $g \cdot m=m^{\prime}$. Note also that for each $\bar{z}_{i} \in Q, \quad \pi_{\bar{z}_{i}}(g)$ is an invertible element of $K_{\left|\bar{z}_{i}\right|}$, since its determinant is $\prod_{j=1}^{|r i|} a_{i j}^{\prime} \neq 0$. Hence by Lemma $0.2, g$ is invertible in $I(Q)$.

Define $\rho: L \rightarrow L^{\prime}$ by $\rho(f)=f^{*} g^{-1}$, for $f \in L$. Then $\rho$ is an isomorphism between $L$ and $L^{\prime}$ as $I(Q)$-modules, as is readily verified. It follows that there is a unique isomorphism class $\mathscr{L}$ of left ideals of $I(Q)$ such that for all $L^{*} \in \mathscr{L},_{I(Q)} I(Q) / L^{*} \simeq M$. The theorem now follows immediately.

As a simple application of the results in this section, let $Q_{1}=$ $\left\{x_{\imath}\right\}_{i=1}^{n}$, where $x_{i} r x_{j}, 1 \leqq i \leqq j \leqq n$. Then $I(Q) \simeq T_{n}$, the algebra of $n \times n$ upper triangular matrices over $K$, as noted at the end of $\S 1$. Since $I\left(Q_{1}\right) \simeq T_{n}$ has a unique isomorphism class of faithful distributive modules, it follows directly from Theorem 3.5 that $T_{n}$ has a unique isomorphism class of faithful distributive left ideals. Note that the collection of $n \times n$ upper triangular matrices over $K$ with $O$ 's in the last column is an example of such an ideal. As another application, let $Q_{2}=\left\{y_{i}\right\}_{i=1}^{n}$, where $y_{i} r y_{j}, 1 \leqq i, j \leqq n$. Then $I\left(Q_{2}\right) \simeq$ $K_{n}$, the algebra of $n \times n$ matrices over $K$. Since $I\left(Q_{2}\right) \simeq K_{n}$ has a unique isomorphism class of faithful distributive modules (which are exactly the primitive modules), it follows directly from Theorem 3.5 that $K_{n}$ has a unique isomorphism class of faithful distributive left ideals (which are exactly the maximal left ideals.) Note that the collection of $n \times n$ matrices over $K$ with O's in a specified column is an example of such an ideal.

\section{REFERENCES}

1. K. Baclawski, Automorphisms and derivations of incidence algebras, Proc. Amer, Math. Soc., 38 (1972), 351-356.

2. W. R. Belding, Incidence rings of pre-ordered sets, Notre Dame J. Formal Logic, 
14 (1973), 481-509.

3. P. Doubilet, G.-C. Rota and R. Stanley, The idea of generating function, Proc. Sixth Berkeley Sympos. on Math. Statistics and Probability, Vol. II, Univ. of California Press, Berkeley, Calif., 1972, 267-318.

4. R. B. Feinberg, Characterization of Incidence Algebras, Dissertation, Univ. of Wisconsin, 1974.

5. — Characterization of incidence algebras, to appear, Discrete Math.

6. - Polynomial identities of incidence algebras, Proc. Amer. Math. Soc., 55 (1976), 25-28.

7. W. Scharlau, Automorphisms and Involutions of Incidence Algebras, preprint.

Received November 20, 1975. This paper was prepared while the author was a National Academy of Sciences-National Research Council Postdoctoral Research Associate at the National Bureau of Standards, Washington, D.C. 20234. The results presented are contained in the author's doctoral dissertation written at the University of Wisconsin. The author's research was sponsored in part by the United States Army under Contract No. DA-31-124-ARO-D-462.

Clarkson College of Technology 



\section{PACIFIC JOURNAL OF MATHEMATICS}

\section{EDITORS}

RICHARD ARENS (Managing Editor) University of California

Los Angeles, California 90024

R. A. Beaumont

University of Washington

Seattle, Washington 98105
J. DUGUNDJI

Department of Mathematics University of Southern Californı

Los Angeles, California 90007

D. Gilbarg and J. Milgram

Stanford University

Stanford, California 94305

\section{ASSOCIATE EDITORS}
E. F. BECKENBACH
B. H. NeumanN
F. WOLF
K. YosHIDA

\section{SUPPORTING INSTITUTIONS}

UNIVERSITY OF BRITISH COLUMBIA

CALIFORNIA INSTITUTE OF TECHNOLOGY

UNIVERSITY OF CALIFORNIA

MONTANA STATE UNIVERSITY

UNIVERSITY OF NEVADA

NEW MEXICO STATE UNIVERSITY

OREGON STATE UNIVERSITY

UNIVERSITY OF OREGON

OSAKA UNIVERSITY
UNIVERSITY OF SOUTHERN CALIFORNIA

STANFORD UNIVERSITY

UNIVERSITY OF TOKYO

UNIVERSITY OF UTAH

WASHINGTON STATE UNIVERSITY

UNIVERSITY OF WASHINGTON

* * * *

AMERICAN MATHEMATICAL SOCIETY

NAVAL WEAPONS CENTER 


\section{Pacific Journal of Mathematics \\ Vol. 65, No. $1 \quad$ September, 1976}

David Lee Armacost, Compactly cogenerated LCA groups ............. 1

Sun Man Chang, On continuous image averaging of probability measures ...... 13

J. Chidambaraswamy, Generalized Dedekind $\psi$-functions with respect to a

polynomial. II................................... 19

Freddy Delbaen, The Dunford-Pettis property for certain uniform algebras ..... 29

Robert Benjamin Feinberg, Faithful distributive modules over incidence

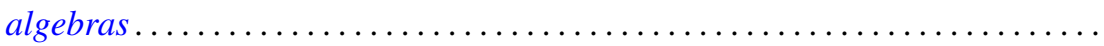

Paul Froeschl, Chained rings . . . . . . . . . . . . . . . . . . . .

John Brady Garnett and Anthony G. O'Farrell, Sobolev approximation by a sum

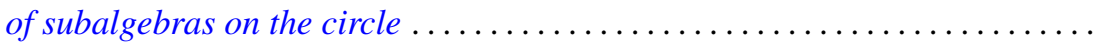

Hugh M. Hilden, José M. Montesinos and Thomas Lusk Thickstun, Closed

oriented 3-manifolds as 3-fold branched coverings of $S^{3}$ of special type.....

Atsushi Inoue, On a class of unbounded operator algebras ................

Peter Kleinschmidt, On facets with non-arbitrary shapes.

Narendrakumar Ramanlal Ladhawala, Absolute summability of Walsh-Fourier

series

Howard Wilson Lambert, Links which are unknottable by maps . . . . . . . . . . .

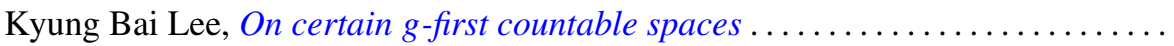

Richard Ira Loebl, A Hahn decomposition for linear maps .................

Moshe Marcus and Victor Julius Mizel, A characterization of functionals on $W_{1}^{p}$ possessing autonomous kernels. I . .

James Miller, Subordinating factor sequences and convex functions of several

variables.

Keith Pierce, Amalgamated sums of abelian l-groups ...

Jonathan Rosenberg, The $C^{*}$-algebras of some real and $p$-adic solvable

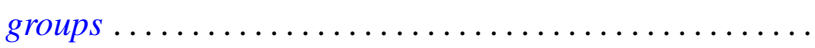

Hugo Rossi and Michele Vergne, Group representations on Hilbert spaces defined

in terms of $\partial_{b}$-cohomology on the Silov boundary of a Siegel domain . .

Mary Elizabeth Schaps, Nonsingular deformations of a determinantal

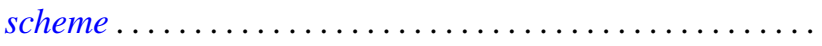

S. R. Singh, Some convergence properties of the Bubnov-Galerkin method...

Peggy Strait, Level crossing probabilities for a multi-parameter Brownian

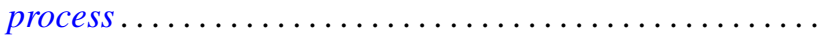

Robert M. Tardiff, Topologies for probabilistic metric spaces .

Benjamin Baxter Wells, Jr., Rearrangements of functions on the ring of integers of

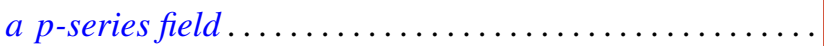

Robert Francis Wheeler, Well-behaved and totally bounded approximate identities for $C_{0}(X)$.

Delores Arletta Williams, Gauss sums and integral quadratic forms over local

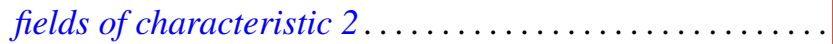

John Yuan, On the construction of one-parameter semigroups in topological 